\title{
Ayllus incas, tierras del sol y agua del Huanacauri en Sucsu Auccaille, San Jerónimo, Cusco
}

\section{Carmen Escalante G.}

(iD) https://orcid.org/0000-0002-6314-4808

Universidad Nacional San Antonio Abad del Cusco atoqescalante@yahoo.es

\section{Ricardo Valderrama Fernández}

Universidad Nacional San Antonio Abad del Cusco

RESUMEN

Tratamos acerca de la transformación del grupo social de Sucsu Auccaille de tener un tipo de organización socioeconómica y politica bajo el Estado inca, a pasar bajo el dominio español y usar documentación escrita y legislación española para defender su posesión sobre la tierra y mantener su unidad como grupo social, dentro de ello el papel que juegan los descendientes de incas que fueron gobernantes. Es una historia de su propiedad de tierras, sus luchas y su continuidad. Nos basamos en diez documentos coloniales, referidos a terrenos de la actual comunidad campesina de Sucsu Auccaille, y en entrevistas a los comuneros, directivos y exdirectivos de Sucsu Auccaille, de San Jerónimo. La etnia inca estaba compuesta de varios ayllus o linajes, dos de ellos eran Sucsu y Auccaille. A inicios de la colonia, estos dos son reducidos en San Jerónimo ${ }^{I}$. Sucsu, la panaca o linaje del inca Huiracocha, y Auccaille, la panaca o linaje del inca Yahuar Huaccac. Dentro de su territorio se encuentran los lugares sagrados Tuynu, Osccollo, Andamachay, Aqhamana, las tierras del sol y el riachuelo Huanacauriue; aún

\footnotetext{
1 Otra parte de Sucsu Auccaille está en San Sebastián.
} 
se cuidan, con sigilo y veneración (a la madre tierra y al padre montaña Huanacauri).

Palabras clave: incas, ayllu, comunidad campesina, propiedad de tierras, Sucsu, Auccaille.

\section{Inca Ayllus, lands of the Sun and water of Huanacauri in Sucsu Auccaille, San Jerónimo, Cusco}

\section{ABSTRACT}

We deal with the transformation of the social group of Sucsu Auccaille, from having a type of socio-economic and political organization under the Inca State, to passing under Spanish rule and using written documentation and Spanish legislation to defend its possession over the land and maintain its unity as a social group. Within it, we are also interested in the role played by the descendants of the Incas who were rulers. It is a story of their land ownership, their struggles, and their continuity. We rely on ten colonial documents, referring to lands of the current peasant community of Sucsu Auccaille, and on interviews with community members, directors, and former directors of Sucsu Auccaille, in San Jerónimo. The Inca ethnic group was composed of several ayllus or lineages, two of them were Sucsu and Auccaille. At the beginning of the colonial era, these two were reduced in San Jerónimo: Sucsu, the panaca or lineage of the Inca Huiracocha, and Auccaille, the panaca or lineage of the Inca Yahuar Huaccac. Within its territory are the sacred places Tuynu, Osccollo, Andamachay, Aqhamana, the lands of the sun and the Huanacauriue stream; they are still cared for, with stealth and veneration (to mother earth and father mountain Huanacauri)

Keywords: Incas, ayllu, peasant community, land ownership, Sucsu, Auccaille. 


\section{AYLLUS INCAS Y COMUNIDAD CAMPESINA SUCSU AUCCAILLE}

La comunidad campesina Sucsu Auccaille de San Jerónimo se encuentra en la zona preínca llamada Oma, donde vivían en vecindad con los sañu, los ayarmacas ${ }^{2}$, los sahuasiras, poqes, lares, alcahuizas, hasta que llegaron los incas, quienes, según el relato mítico, bajaron del cerro Huanacauri ${ }^{3}$. Los primeros incas hicieron alianzas tomando esposas de los grupos originarios. Así, Sinchi Roca Inca se casó con la hija del señor de Sañu y Lloque Yupanqui se casó con la hija del señor de Oma 5 . Segun las crónicas, la segunda alianza matrimonial de los incas en el valle del Cusco es la realizada entre este grupo y la etnia oma, hoy San Jerónimo. Entrar como yerno a un grupo social andino tenía sus especificidades. El yerno tiene un conjunto de roles prescritos, de servicio y obediencia a su suegro y a la familia de este, como si fuera su padre. Igualmente, el suegro debe protección y ayuda a construir su familia a su yerno como si fuera su verdadero hijo. Establecen una relación de ayuda mutua y lealdad.

La comunidad Sucsu Aucaille lleva el nombre de dos panacas o linajes incas: Sucsu, de Huiracocha Inca, y Auccaille, de Yaguar Huacac Inca. En los documentos, a partir de 1565 se denomina Sucsu panaca y Auccaille panaca, y están juntos como un grupo compuesto, y luego, en la República pasa a ser denominada comunidad campesina Sucsu Auccaille, según las leyes vigentes.

\section{Ayllus incas}

El término ayllu designó a una organización social inca basada en lazos de parentesco, origen común y propiedades comunes, como estar vinculadas a un

\footnotetext{
2 Tengamos en cuenta que, actualmente, todo un barrio del pueblo de San Sebastián es ayarmaca, del mismo grupo étnico que habitaba una extensa zona del Cusco antes del señorío local de los incas.

Según su mito de origen.

Según lo relatado a los cronistas.

Según lo relatado a los cronistas.
} 
territorio. La referencia en documentos a Sucsu Auccaille es como ayllu, a veces panaca, o el uso de ambos términos. Panaca, definido por Sarmiento de Gamboa, es linaje, grupo de parientes.

Según informaron los Incas a Sarmiento de Gamboa, las panacas eran:

\section{Cuadro 1. Panacas de Hurin y Hanan Cusco}

\begin{tabular}{ll}
\hline \multicolumn{1}{c}{ Panacas antiguas } & $\begin{array}{c}\text { Similar actual en el distrito de San } \\
\text { Jerónimo }^{6}\end{array}$ \\
\hline Chima Panaka (Manku Qhapaqpa panakan) & Ayllu Chima \\
Rawrawa Panaka (Sinchi Ruq'ap panakan) & Ayllu Raurahua \\
Hawaynin Panaka (Lluq'i Yupankip panakan) & \\
Uska Mayta Panaka (Mayta Qhapaqpa panakan) & \\
Apumayta Panaka (Qhapaq Yupankip panakan) & Ayllu Apumayta \\
Wikakiraw Panaka (Inka Ruqap panakan) & A Roquequirao o Vicaquirao \\
Awqaylli Panaka (Yawar Waqaqpa panakan) & AylluSucsu-Aucaille \\
Suqsu Panaka (Wiraqucha Inkap panakan) & Ayllu Sucsu-Auccaille \\
Hatun Ayllu (Pachakutiq Yupankip panakan) & Ayllu Anahuarqui \\
Qhapaq Ayllu (Tupaq Yupankip panakan) & \\
Tumipampa Ayllu (Wayna Qhapaqpa panakan) & \\
\hline
\end{tabular}

Esta relación de panacas es aceptada casi sin mayor cuestionamiento, pese a haber ciertas discrepancias en torno a la existencia de la última panaca, así como a la integración —o no- de los dos grupos asociados con Pachacútec: Iñaca Panaca y Hatun Aillu. Para el estudioso Tom Zuidema se trata, más que de una sucesión de linajes incas, de una estructura de organización social. Estos grupos coexistían. La mayoría de crónicas se refieren a doce incas sucesivos; sin embargo, la crónica de Fernando de Montesinos da una relación de cien incas jefes del gobierno del Tahuantinsuyo, los diez últimos denominados incas (Szeminski, información personal). El término panaca se usó para designar un grupo social o ayllu de

6 Siete ayllus coinciden con el nombre de las antiguas panacas, los otros son: Ayllu Accamana, Ayllu Antamachay, Ayllu Surama, Ayllu Orqon, Ayllu Halampata, Ayllu Qollana, Ayllu Qollancas, Ayllu Chahuan Qosqo.

7 Lo vinculan con Pachacutec por haberse este inca casado con la mujer llamada Anahuarqui.

8 El nombre de los once ayllus es tomado de Hernández Astete (2008). El añadido en cursiva es nuestro. 
parientes de un inca, en el sentido de descendientes. Además de funcionar como un ayllu cumplían la función de cuidar la momia de su ascendiente, realizando ceremonias y rituales, para lo cual poseían ganado, cocales y tierras específicas para producir los productos que destinaban al culto y memoria de su antepasado. Sarmiento de Gamboa dijo que panaca significa descender:

Hizo el primer ayllu y llamole Chima panaca Ayllo, que quiere decir linaje que desciende de Chima; porque el primero a quien dejó encomendado su linaje o ayllo se llamó Chima, y Panaca quiere decir descender (Sarmiento, 1988, pp. 63-64).

Los ayllus Succsu y Auccaille se encontraban asociados ya en tiempos de los incas; así, Cristóbal de Molina escribió hacia 1575 la forma como participaba la nobleza cusqueña en la fiesta del citua:

\section{Cuadro 2. Panacas y los cuatro suyos}

\begin{tabular}{cccc}
\hline \multicolumn{2}{c}{ Rurin Cusco } & \multicolumn{2}{c}{ Hanan Cusco } \\
Contisuyo & Collasuyo & Antisuyo & Chinchaisuyo \\
\hline Chima Panaca Aillu (1) & Ahuaymin Aillu (3) & Aucailli Aillu (7) & Vicaquirao Aillu (6) \\
Yaura Panaca Aillu (2) & Uscamaita Aillu (4) & Suzso Panaca Aillu (8) & Hatun Aillu (9) \\
& Apomayta Aillu (5) & & Ccápac Aillu (10) \\
\hline
\end{tabular}

Fuente: Hernández (2008, p. 36).

Max Uhle, en su obra Estudios sobre la historia incaica (1969, p. 19) también asocia Auccaille y Sucso al Antisuyo. Lo interesante es que lo que une desde esa época a Auccaille y a Sucsu no solo es que descienden de Yaguar Huacac, sino que ambos son considerados del Antisuyu'.

En 1533, con la entrada de los españoles a la ciudad del Cusco, despojaron de sus propiedades a los descendientes de incas y los redujeron a poblaciones de la frontera de la ciudad, a pueblos como San Sebastián, San Jerónimo, Chincheros, Maras, y a los barrios de San Cristóbal, Santa Ana y Santiago. El inca que colaboró con los conquistadores españoles, el hermano de Manco Inca llamado Paullu Inca, vivió hasta su muerte en el barrio de San Cristóbal. La Iñaca panaca, que

9 El estudio genético realizado por José Sandobal y Ricardo Fujita, de la Universidad San Martín de Porres, confirma que las personas agrupadas actualmente en Sucsu Auccailli tienen un ascendiente común. 
corresponde al inca Pachacutec, tuvo sus tierras en San Jerónimo. Sarmiento de Gamboa cuenta que el inca Pachacutec:

refundió a Iñaca panaca con Hatun ayllu en uno solo, pero nunca se realizó dicha unión porque ambos siguieron existiendo en el Cusco colonial [...] Durante el virreinato, Iñaca panaca pertenecía a la parroquia de San Jerónimo y en una provisión hecha en el Cusco en 1630, sobre deslinde de heredades, las tierras de Iñaca panaca eran vecinas, por un lado, con las de los ayllus de Sucsu y Auccaylli ${ }^{10}$, y en otro lugar, con los campos de los Chauin Cusco y Arayraca (ACC, legajo 8, cuaderno 22, año 1713) (Rostworowski 1983, p. 144).

En el documento n. ${ }^{\circ} 2$ (1647) de reparto de tierras a Sucsu Auccaille, citan:

Palcanca. Les di la posesión de las tierras del asiento de Palcanca que están con linderos y moxones con el río de Huanacauri y con tierras del convento de la Merced y linde de Iñaca panaca Chauin Cusco y Arairaca. Por ambas partes y los dos de ellos dichos dos ayllus Sucsu Auccaille hay veinte 20 topos.

$\mathrm{Su}$ existencia en los archivos nos muestra que era una panaca realmente existente. Otra mención en el mismo documento n. ${ }^{\circ} 2$ es:

Poma orco. Les di posesión de las tierras del asiento de Poma orco que lindan con el río de Mama tunya Guaico y por el otro lado de Iñaca panaca que está en medio que tienen ambos ayllus, cincuenta topos de sembrar trigo y maíz de regadío que ellos lo dixeron.

En el mismo documento n. ${ }^{\circ} 2$ se menciona:

Conopampa Guayllapampa - Les di posesión de las tierras del asiento de Conopampa guayllapampa que lindan con Iñaca panaca y de los yanaconas de la dicha parroquia que dijeron tenían y poseyan de su naturaleza quarenta topos entre ambos ayllus.

En 1559, Polo de Ondegardo, en un afán de ordenar la mano de obra, reúne a unos 20000 indígenas de la ciudad del Cusco y los asigna a pueblos de alrededor de la ciudad. En 1571 Toledo visita la ciudad del Cusco y ordena formalizar y crear las ocho parroquias existentes aún hasta la actualidad. Aunque teóricamente eran parroquias de indios, dentro de su espacio vivían españoles, criollos, mestizos y aún algunos negros. La parroquia de San Jerónimo se funda, siendo sus caciques

10 En los documentos de 1595 y 1630, se menciona a Iñaca panaca como colindante de Sucsu Auccaille. 
Pascual Tupayupanqui panaca ${ }^{11}$ y Pascual Quispe Rimachi. Se alza en el lugar donde había una huaca Inca. Utilizan en su construcción piedras con litograbados de una antigüedad de 5000 años a. C.

En 1572, en el pueblo de San Jerónimo de Oma, se reducen los ayllus de: Ayllu Sorama (originario de urin), Urin Ccoscco, Yabacona, Collana, Urin Saca Mama y Chimaraurau (Chima panaca de Manco Capac Inca de Urin, Raurau panaca de Sinchi Roca)). Durante la colonia se menciona los ayllus: Yanacona, Ccollana Chawan Qosqo, Ccallampata, Urin Aqha, Mama Chimaraura, Aqhamana, Apumayta (panaca de Capac Yupanqui), Roqhe quiraw, Rau rau (panaca del Inca Sinchi Roca), Orqon puquio (Picol Orqonpugio), Sucsu Aucaylli (panacas de Huiracocha y Yaguar Huacac), Kirkas, Qonchacalla. Sorama. Urin Qosqo. Hasta la actualidad existen estas catorce comunidades en la población de San Jerónimo.

\section{CEQUES, AYLLUS Y COMUNIDAD SUCSU AUCAILLE}

Las tierras de Chima panaca, descendientes de Manco Capac, se encontraban en la cuenca del río Huancaro, en lo que son hoy las comunidades de Chocco y Cachora. El cronista Bernabé Cobo dice que esta panaca cuidaba el quinto ceque, los lugares religiosos que empezaban en el mismo Coricancha, el lugar denominado Caritampucancha, luego Tiucalla, en Cayacache, lugar en el cual había unos guerreros de piedra. El tercer lugar era Cayallacta, un grupo de piedras en el cerro de Choco. La cuarta era Churupuquiu, un manante en el cerro de Choco. La quinta era Cumpu Guanacauri. Cumpu es abultamiento y Guanacauri es el nombre del cerro, del Apu y segundo oráculo en la época del Tahuantinsuyo. Chima panaca es uno de los grupos que fueron reducidos en San Jerónimo.

La panaca de descendientes de Lluq'i Yupanqui, inca que se casó con la hija del señor de los Oma, se especializó en ser sacerdotes o tarpuntaes. Y cuidaban el tercer ceque que iba al Collasuyu. Este empezaba en Limac Pampa, lugar plano en el cual se realizaba la fiesta del maíz. La segunda era Raquiancalla, una elevación en la cual había huacas. La tercera, Sausero, un maizal en el cual se iniciaba ceremonialmente la siembra. La cuarta, Oma talispacha, un manantial que se adoraba. Oma en puquina significaba «agua». La quinta, Oscollo, un terreno llano, actualmente existe Oscollo pampa. La sexta, Tuynu Urcu, grupo de piedras, cerca de la granja de Kayra. La séptima, Pallpanccay puquio, un manantial cerca

11 Aquí el término panaca está usado como rango de autoridad y ciertamente no se encuentra después como apellido en ningún otro integrante de la comunidad. 
de la granja de Kayra. Según el cronista Bernabé Cobo a esta huaca le ofrecían mullu (conchas) molidas. Con el mismo nombre del manante se nombra a las tierras y en la época inca eran "las tierras del sol". Actualmente existe, ese sector se denomina Pallpanccay. La octava huaca o adoratorio era Ccollocalla, un mojón o marcación de límite en una quebrada. Ccollo en quechua significa que no se realiza o improductivo, infértil y calla es comenzar. Comienzo de la improductividad. Estas huacas están en terreno del actual distrito de San Jerónimo, dado que los descendientes de Lluq'i Yupanqui eran descendientes también del señor de los omas, por el lado materno, tal vez por ello les adscribieron como cuidadores de estas Huacas del tercer ceque, la mayoría de las cuales estaban en territorio oma.

En este tercer ceque, camino al Collasuyo, y tercera huaca llamada Sausero, para el día de la siembra de maíz, participaba el mismo inca y salían del Cusco con sus momias portadas por sus respectivas panacas y los nobles del Cusco, con doscientas acllas, y empezaban con la alabanza al sol. Cuando este se alzaba, cantaban alzando la voz, y cuando bajaba, bajaban la voz del canto. Este ritual se hacía en Sausero, con participación del inca, tanto en siembra como en cosecha. El inca bebía chicha y comía maíz con los sembradores.

\section{TIERRAS DE LA COMUNIDAD SUCSU AUCCAILLE}

Las tierras de Sucsu Aucailli son reconocidas en documentos desde inicios de la colonia. Presentaremos varios de ellos. Primero tenemos en su Título de $1595^{12}$, que consigna la repartición de pasto y ejidos, realizado por el juez visitador capitán don Francisco de Loayza en el año de mil quinientos noventa y cinco (1595) a fojas 158 , hasta fojas 160 .

El dicho visitador declaró y dejó por pastos y egidos de esta dicha Parroquia todas las tierras de guaylla que hay desde el mojón y pared de adobes que se dejó y puso por señal, por bajo y junto a la barranca que hacen las tierras de Runcu guasi, corriendo el largo de las dichas tierras de guaylla, por delante de las tierras llamadas Mitcopampa y van corriendo por delante de esta dicha Parroquia el Camino Real abajo hasta llegar a los huecos del molino del Convento de Santo Domingo y desde el dicho molino van corriendo las dichas tierras y pasto por la gialla, dejando el Camino Real sobre mano derecha, como se va a la Angostura hasta llegar al río que viene de la estancia de Cayra. En que habrá mas de cuarenta. Les dio veinte topos en Copampa, y diez topos en Ptoinocuchu.

\footnotetext{
12 Archivo de la Comunidad Campesina Sucsu Auccaille (en adelante, ACCSA), fols. 158 a 160.
} 
Notese que el documento anterior menciona los guaillares y pastos que pueden usar los integrantes de Sucsu Aucailli.

Tenemos además un segundo documento de $1630^{13}$ que reconoce las tierras de sembrar, sobre todo maíz y trigo:

El protector de naturales 1638 se presentó esta petición: $=$ el protector de los naturales en nombre de Don Pedro Antón y Don García Topa Atauche Caciques de los indios de Sucsu y Auccaille Ynga de la Parroquia de San Jerónimo $=$ digo como consta de esta executoria Real del que hago demostración ganada en contradictorio juicio por ser tales Yngas verdaderos están reservados de tasa y tributo y de servicios personales". "digo como consta y parece por esta provisión Rreal emanada de las Real Audiencia de los Reyes $\mathrm{y}$ obedecida por los jueces oficiales reales, en que mandaron volver y restituir las Don García Topa Atauche y Don Pedro Antón Atayupanqui y Don Sebastián Poma Supa Yngas, caciques y gobernadores de los ayllus Sucsu y Auccaile de la generación de Viracochán Ynga, Yaguar Vacac Ynga Señores que fueron de esta tierra rreducido en la Parroquia de San Jerónimo tierras de la rrepartición que tienen poseen en los asientos de, Cayrapampa, Condenauqui, Colparu pampa, Palpamcay pampa, Guacapongo, Picocaipampa, Cozcopampa, Copampa y Quinchacaira Tinyacucho, Colpampa, Chuncuran yami, cullulaqta, Siusacucha, Totora villa, Pomaguanca, que están asentadas en el libro de la dicha rrepartición con sus mojones y linderos y los dichos señores presidentes e oidores por la dicha su provisión mandase sean restituídos en ellas $[\ldots]$

\section{TIERRAS DEL SOL. SUCSU AUCAILLE}

En $1691^{14}$ hay un tercer documento, sobre un litigio interpuesto por Guillermo de Balladares y Esquivel, sobre 18 topos de tierra llamados Osccollo pampa, que dice fueron de los padres de su esposa Gerónima Fernández Escudero y que le entregaron como dote, contra «los Caciques y común de los ayllus Sucsu y Aucaille reducidos en la Parroquia de San Jerónimo y de los ayllus Sañus de Tarpuntay de la Parroquia del Señor San Sebastián», para lo cual revisan el documento que:

hizo el Capitán Francisco de Loayza, vecino y Juez de naturales, de repartición y acomodamiento de las dichas tierras [...] 1595 [...], la repartición de tierras de los ayllus de Sucsu panaca y Auccaille panaca. De la encomienda de Melchor Vásquez de Dávila, la que sacada a la letra es como sigue: [...]

\footnotetext{
13 ACCSA, fol. 15.

14 ACCSA.
} 
Don Alonso Tito Atauche un topo de Pallpanccay. Un topo en la segunda suerte de Cairapampa, Uno y medio topo en Guaca pongo, que a derecho ser suyo y heredero del Guayna Capa por ser su bis nieto, un topo en Collparo huqui, un topo en Picoraipampa, un topo en Uscollopampa, un topo en Copco pampa, medio topo en Guayllapampa, un topo en Oscollopampa.

Francisco Quesso. Un topo en Pecoraypampa, un topo en Quillairo pampa, un topo en Usccollo pampa.

Juan Pichota. Dos topos en Halpa corcona, cuatro topos de tierra de andenes en Pallpancay que fueron del sol y medio topo en Guayllapampa.

-Gerónimo Puso mayo. Un topo en Pallpanccay, dos topos en Cairapampa, un topo en Quillairo, un topo en Comopuro ${ }^{15}$.

Pallpanccay es un sector de terrenos de cultivo que aún queda dentro de la comunidad de Sucsu Auccaille, sector que es regado con agua del riachuelo Huanacauri y que en este documento dice claramente que «fueron tierras del sol». Las tierras del sol estaban destinadas a ser cultivadas para realizar las ofrendas a la deidad, así como para mantener a los sacerdotes y acllas. Sus productos se guardaban en depósitos y en caso de necesidad se recurría a ellos para dar alimento a regiones afectadas por sequías, friajes, o bien desastres a causa de abundante lluvia. El documento es una prueba de cómo los españoles y criollos iban despojando de sus tierras a los Incas, y estos se encontraban en una constante lucha por defender la poca tierra que les iba quedando. Ya en 1595, encontramos diferencia en cuanto a la cantidad de tierras que poseía cada comunero. Alonso Tito Atauche poseía ocho topos de tierra, Juan Pichota seis y medio, Gerónimo Puso Mayo cinco topos y Francisco Queso tres topos. Vemos que existe diferenciación en la posesión de tierra de cultivo.

\section{USHNUY AGUA DEL RÍO HUANACAURI O GUANACAURE MAYO}

Parte del tercer documento de 1691 continúa describiendo las posesiones de Sucsu Auccaille, denominándola «panaca» y también se refiere al «Ushnu» (lugar donde se filtra líquidos), cuya función básica es la de conectar el mundo físico, el de «aquí», con el mundo de las deidades, y por ello se coloca en el ushnu las ofrendas y se supone que los dioses las reciben. Zuidema (1974/76, pp. 199-230) explicaba que ushnu esencialmente es un lugar de drenaje de líquidos, no solo es

\footnotetext{
15 Tercer documento. ACCSA.
} 
lo que conocemos como plataformas superpuestas, sino también pozos o canales. El ushnu de Sucsu Auccaille es de plataformas superpuestas, actualmente desmoronadas. En el documento se menciona el lugar llamado Ushnu:

Razón de las tierras de papas y punas, alboradas y agua que tienen estos indios de ayllu Sucsu panaca de estas dichas parroquias: Punas.- las tierras de puna que se hallaron visitaron a estos dichos indios de Sucsu son desde un cerro llamado Tintir y va subiendo a otro cerro llamado Ushnu ${ }^{16}$ donde hay unos paredones y linda y es de mojón con tierras de los indios de Uroyacamana ${ }^{17}$ y de allí va a dar a un guaico llamado Yaollipongo y de allí va a dar a otro cerro llamado Cuicuhuisa y de allí bajando a un río Yamonoya que es un pueblo viejo y de allí sube a un guaico llamado Atpitán y de ahí bajan a Pacchaicoc y de allí bajan a Marca pongo y Munay guarmi, las cuales dichas tierras el dicho visitador dio y adjudicó en lo que les pertenece [...] arboledas $[\ldots\}$ las arboledas que se halló y visitó a estos indios en Comillonca guaico, les daba y dio a los indios [...] las aguas [...] el dicho Visitador dijo que les daba y dio comisión a estos dichos indios para que usen y guarden la costumbre antigua que han tenido y tienen en regar las tierras que les han sido dadas y repartidas con las aguas de Quiquijana Guaico y con las de Caira mayo. Y con las de Micay puquio y con las de Piap [sic] Guayllanpuquio y de Quiguarpuquio y que en dicho riego guardan la costumbre que los Incas dejaron... Agosto/22/1595. G/abril/1647/.

Tan importante como las tierras eran (y son) las aguas para regarlas, y en este documento es bien claro con qué agua riegan.

En otro documento se menciona también el arroyo de Guanacaure, o bien Guanacaure mayo, el cual siguen poseyendo hasta ahora.

y con el cerro y arroyo de Guanacaure ${ }^{18}$ mayo y de Tarpuntay Sañoc, donde tienen ambos ayllus Sucsu y Auccaille setenta y cinco topos ${ }^{19}$.

En el documento de 1691, se reconoce su derecho al uso de sus fuentes de agua y de que «en dicho riego sigan la costumbre que los Incas dejaron» ${ }^{20}$,

16 Ushn es la denominación que dan al lugar sagrado donde ponían las ofrendas para las deidades en época de los incas.

17 Acamana es el antiguo nombre del Cusco; los incas ya encontraron este lugar llamado así. Actualmente es el nombre de una de las montañas que está en la margen derecha de la ciudad del Cusco.

18 El cerro Guanacaure en tiempos de los incas fue el segundo oráculo mas importante después del Qori cancha.

19 Copia de documentos del Archivo Departamental del Cusco. ACCSA.

20 Documento de 1691. ACCSA. 
haciendo referencia a la Visita de 1595. El último párrafo es muy significativo, por cuanto supone el tipo de reparto de agua de riego que tenían de acuerdo a la «costumbre» y también los rituales que debieron hacer a lo largo del tiempo.

\section{PARENTESCO}

No solo en un cuarto documento de $1663^{21}$, sino también en los anteriores, se detalla a los poseedores de los terrenos y se puede ver el parentesco entre ellos, así como sus apellidos que son similares a los apellidos de los actuales comuneros. El análisis de sus actividades es que los primeros incas de Sucsu Auccaille eran guerreros, diplomáticos, políticos, administradores, constructores como Juan Felipe Francisco Conde Ynga Senche Ruca Inca Capac. A inicios de la República aun ocupaban cargos públicos. En inicios del siglo XX tenemos mas campesinos y surgiendo los comerciantes. Los actuales son comerciantes, transportistas, empresarios y profesionales, profesores, ingenieros, abogados, y por supuesto, antropólogos e historiadores.

En los «Títulos de Propiedad de la Comunidad Sucsu Aucaille» se lee:

de todas aquellas tierras, tocantes al dicho Señor Don Diego Senche Ruca Ynga Cacique Gobernador de la ciudad del Cusco que fue de la parroquia de San Gerónimo, del ayllo de Sucsu Aucaille que fue nieto legítimo heredero de las dichas tierras contenidas en esta Real Provisión del Rey mi Señor, y no poderlas repartir a los indios ni vender a otra persona, ni comprar porque son tierras mayorrasguías desde el tiempo de la antigüedad de la primera conquista ganó las dichas fanegadas de tierra, [...] Lima - Enero de mil seiscientos noventa y cinco años de $[1695]^{22}$.

... a los principales de la parroquia de San Gerónimo del Ayllu Sucsu Auccaille que dueño de las tierras contenidas en la dicha real posesión de amparo de tierras en la dicha real hacienda del Capitán Don Diego Senche Ruca Ynga, de mil quinientos setenta y nueve años.- título del muy nuestro Señor Don Felipe Quinto.

En este mismo legajo se transcribe otro documento de 1633:

En la dicha ciudad del Cusco en veintitres días del mes de agosto de mil seiscientos treinta y tres años. El Capitán don Francisco Castillo de Altamirano, Gobernador de la ciudad del Cusco.- El protector general de los naturales de

\footnotetext{
21 ACCSA.

22 Cuarto documento de 1695. ACCSA.
} 
la ciudad del Cusco, distrito en nombre del Señor Don Diego Senche Ruca Ynga, hijos, nietos lergítimos, de Juan Felipe Francisco Conde Ynga Senche Ruca, [f.4 ] Inca Capac que fue su bisabuelo materno alcanso desde España un decreto del Señor Rey Don Carlos Quinto ${ }^{23}$ [que Dios guarde] etc. Y como sus herederos como consta de estos recaudos y amparo de las tres fanegadas y media de tierras de sembrar maíz y trigo en el asiento y lugar llamado "Tutuna pampa" confina las tierras de Tuyno y unas dos fanegadas y media de tierras en el asiento y lugar llamado Quencha guaico, [...] Ampare al dicho Don Diego Senchi Ruca Ynga Cacique Gobernador de la Parroquia del Señor San Jerónimo, del ayllo Sucso Aucaille ${ }^{24}$, se menciona también a Pedro Sinchi Roca.

La familia Sinchi Roca mencionada en el documento aún se cuenta entre los comuneros en Sucsu Auccaille. Como prueba de que son descendientes directos de los Sinchi Roca ${ }^{25}$ mencionados en documentos coloniales. Los descendientes de esta familia poseen los documentos de las probanzas que presentaron sus antepasados, documentos que han heredado de ellos. De esta familia, los primeros ancestros mencionados de la época de 1544 son: Juan Felipe Francisco Conde Ynga Senche Ruca, [f.4 ] Inca Capac que fue bisabuelo de Diego, don Alonso Sinche Ruca Ynga Tito Yupanqui y Josep Sinchi Ruca Tito yngas, quienes obtuvieron un decreto del rey don Carlos Quinto dice que los «tengan siempre ocupados en oficios y públicos en la ciudad del Cusco y parroquia de San Jerónimo» ${ }^{26}$.

Aparte de haber participado en la conquista de Potosí, al lado de los españoles, también se menciona que Sinchi Ruca, luego de luchar contra los atahuallpistas junto a Francisco Pizarro, fue capitán de una de las compañías que se formaron para combatir a Gonzalo Pizarro, combatió junto al presidente La Gasca, y también como era costumbre esa época él mismo puso su gente o soldados, las armas y el sustento para su batallón, como reitera en su documento no le costó nada a Su Majestad. Él mismo es capitán de esa compañía, para luchar contra el rebelde Gonzalo Pizarro, participa en la batalla de Xaquixahuana, donde derrotan a Gonzalo Pizarro, lo aprehenden, lo juzgan, lo sentencian y lo decapitan.

23 El rey Carlos I de España y V del Imperio Romano Germánico vivió entre 1500 a 1558. En 1544 reconoció a la mayoría de descendientes de incas que habían ayudado a los españoles en la conquista del Perú.

24 Documento de 1633. ACCSA.

25 Ronald Elward ha investigado sobre los actuales Sinchi Roca, encontró en archivos las partidas de bautismo, las de matrimonio de sus padres, abuelos, y así llegó a lo más remoto posible. Un avance de su investigación está publicado en el dominical de El Comercio de Lima, en septiembre de 2012, «Los Sinchi Roca: el poderoso vínculo con la tierra».

26 Foja 9 del Documento del Archivo Privado de Octavio Sinchi Roca. 
En cierta forma, con esta victoria sobre Gonzalo Pizarro, venga la muerte de los suyos. Estos yngas del siglo XVI tenían por oficio ser guerreros y políticos; a la llegada de los españoles lo siguen siendo, eso es lo que sabían hacer. Como en el caso, ya estudiado de Paullo Inca, Don Alonso Sinche Ruca Tito Ynga ayuda a los españoles en la conquista del Collao:

sacado de los originales y pasado por el real consejo de yndias y autorisada Don Francisco Hernandez de Madrigal Oficial Mayor que fue de la secretaria del Perú con esta que el dicho Don Alonso Sinche Ruca Tito Ynga nieto principal del gran Ynga Sinchi Ruca fue de balor y gran guerrero y conquistó la gran provincia del collao y después del pueblo de Maon por los franceses y ganó la batalla de Bajo de la Corona Real de Armas las del Rey Nuestro Señor que Dios Guarde. [...] el que asistió a los recuentros y batallas [...] y pacificaron a estos reynos ${ }^{27}$.

Como en el caso de Francisco Tupa Guaman Rimachi ynga (Escalante y Valderrama, 2014), que es testigo de la entrada de Manco Inca a la Plaza de Cusco acompañado de los primeros españoles y cuya hermana fue mujer y madre de la hija de Gonzalo Pizarro, igualmente don Juan Felipe Francisco Sinchi Ruca ynga acompaña a los hermanos Pizarro en Cajamarca, luego lucha junto a La Gasca y es capitán de un batallón contra Gonzalo Pizarro. Estos méritos a favor de la Corona española le valen tener ciertos privilegios.

y al dicho Don Felipe Sinche Ruca Tito ynga así mismo su bisabuelo materno hizo merced el Señor Emperador Carlos Quinto por provisión de primero de octubre de mil y quinientos cuarenta y quatro ... los hijos e hijas que al presente indios Don Sebastiana Ruca ynga hermana legítima de Felipe y Carlos y Fernando Sinchi Ruca Yngas"28 a quienes alcanza las mercedes concedidas por el Rey en 1544.

Luego (Doc.4) se cita a su bisnieto (de Juan Felipe Francisco Conde Ynga Senche Ruca Inca Capac), el cual es Diego Sinche Ruca Ynga, mencionado en 1663. Presentó Probanza en $1655^{29}$, en la cual recuerda los servicios que prestaron al rey de España sus ascendientes. Su descendiente fue Lorenzo Senchi Roca. Don Diego Siche Ruca Ynga fue hijo legítimo de Alonso Sinche Ruca Ynga y de doña Pascuala Sinche Ruca ñusta coya. Como un paréntesis aclaremos que

\footnotetext{
27 Archivo Privado de Octavio Sinchi Roca.

28 Archivo Privado de Octavio Sinchi Roca.

29 Elward (2012) presenta el árbol genealógico de descendientes en línea directa de Diego Senchi Ruca.
} 
el término coya se usaba para la esposa principal del Inca, la cual debía ser su hermana de padre y madre (el caso que hemos estudiado en las crónicas es cuando Huáscar pide a su madre biológica que le dé una hermana para su mujer y poder entronizarse como Sapa Ynca a la muerte de Huayna Ccapac). El término palla se usaba para una mujer noble, también para una esposa del inca que no fuera la principal y el de ñusta para una mujer noble. En los documentos coloniales de la familia Topa Guamanrimachi Ynga, usan el término ñusta al final del nombre de la esposa.

En este documento se hace referencia a fray Domingo de Cabrera y Lartaún, muy recordado en el pueblo, inclusive hay una calle que lleva su nombre:

En este documento se ve la importancia de don Diego Sinchi Roca, sus ascendientes, su bisabuelo Juan Felipe Francisco Conde Ynga Senchi Ruca Inca Capac, su padre Alonso Sinche Ruca Ynga, así como sus descendientes, como Lorenzo Sinchiroca, nacido en 1745 y Alférez Real en 1805, y los de Gaspar Tupac Yupanqui, Felipe Tupac Yupanqui y Pedro Orccohuarancca. Actualmente, existen las familias Sinchi Roca y Tupac Yupanqui en Sucsu Auccaille, e inclusive comuneros que se apellidan Sinchi Roca Tupacyupanqui. Un recibo de $1701^{30}$ confirma esta relación de parentesco, así como el uso de dinero.

Digo yo Doña Manuela Senchi Ruca, ñusta de la ciudad del Cusco que fue de la parroquia de mi Señor San Jerónimo, del ayllu de Sucsu Aucaille, que es verdad que yo recibí cuarenta y ocho pesos de plata contada que ha dado el Señor Don Felipe Conde y Hurtado, caballero Ynga del Orden de Santiago Gobernador y Capitán G. -/f. (sic) que fue Alférez Real de las ocho Parroquias de la infantería de los nobles que vive en la Parroquia del Señor San Blas por lo que me recibo en su mano unos título originales de tierras llamadas Quencha quencha, Pata-pata guayco de sembrar maíz y trigos y las pampas (sic) también que por todo constan dos fanegadas y media de tierras" Siendo sus testigos: "Don Felipe Chiguantito, Don Andrés Topa Ynga, Don Diego Orcco guaranga Ynga ${ }^{31}[\ldots]$ Los apellidos Chiguantito y Orccoguaranga continúan existiendo en la comunidad Sucsu Auccaille.

Por su posición, a los varones les llamaban ynga y a las mujeres ñusta, aún en 1700. Las mujeres tenían derecho sobre sus tierras, las heredaban, así como las vendían. En el 1700 el uso de dinero es ya común.

\footnotetext{
30 Quinto documento. ACCSA.

31 Copia de documentos del Archivo Departamental del Cusco. ACCSA.
} 
En un sexto documento de 1701 se menciona también a Lorenzo Sinchi Roca que fue propietario de esas tierras a mediados del $1700 \mathrm{y}$ hasta la primera década del 1800.

En el Testimonio de la excepción de los Yngas (De la Puente, 2016, p. 47) del siglo XVIII, se hace mención a 1584. Pertenece al expediente de doña Tomasa de Medina Guzmán Atau Yupanqui, descendiente de Huiracocha Inga ${ }^{32}$. En él también se habla de las familias de la comunidad Sucsu Auccaille, por ser los Atau Yupanqui relacionados a esa comunidad.

«Declaración que en 16 de enero de $1584^{33}$ hicieron don García Tito Gualpa y otros consortes ante los señores Damián de la Bandera ${ }^{34}$ y García de Melo Jueces comisarios en la causa que siguieron con los indios Yngas Descendientes de los Reyes, Señores que fueron del Perú, en su Gentilidad sobre su Hidalguía exención de privilegios y demás deducidos...» (ARC Colección Betancourt 1584, f. 252-265) (De la Puente, 2016, p. 47).

El noveno documento es la «Probanza de los nietos de Tupa Yupangui señor que fue deste Reyno y de sus hermanos», Cuzco, mayo de 1569 (Rowe 2003 [1985]).

En el documento incluye a los descendientes de tres hijos de Pachacuti de Capac Aillu. Los nietos de Tupa Yupangui fueron organizados en tres ayllus: Qollana, descendientes de Tupa Inca Yupanqui, Pallan, descendientes de su hermano Amaru Tupa, y Callao descendientes de Tupa Yupangui otro hermano de Tupa Inca Yupanqui (De la Puente, 2016, p. 48).

Aunque nuestro estudio comprende a Sucsu y Auccaille, sin embargo vamos a transcribir a los descendientes de incas comprendidos en el Testimonio de 1585, por cuanto muchos de esos apellidos se repiten en la comunidad actual de Sucsu Aucccaille. En el testimonio de la excep[ió]n de los Yngas [f. 158] citado por De la Puente (Séptimo documento), dice:

Por lo que toca a Don Francisco Sayre Topa, y a los demás Yngas del ayllo Sucso descendiente del Viracocha Ynga, señor natural que fue de estos reynos, reducidos en la Parroquia de San Sebastián de esta ciudad. Digo que

\footnotetext{
Su panaca fue Sucsu.

Octavo documento. ACCSA.

Nació en España por 1520, su padre fue uno de los primeros conquistadores de tierra firme. Tuvo una vida azarosa al lado del Demonio de los Andes, y luego luchando contra él a favor del Rey. En 1557, cumpliendo órdenes del virrey y del príncipe don Felipe, escribió su notable «Relación General de la disposición y calidad de la provincia de Huamanga, llamada San Juan de la Frontera, y de la vivienda y costumbres de los naturales della» (Inf. de Wikipedia).
} 
al Derecho de mis partes conviene que se saque uno, dos, o mas traslados que hubieren menester autorizados en debida forma, y manera que haga fe con este testimonio de que ha [f.158v.] go demostración de Luis de Quesada escribano público que fue de esta ciudad (De la Puente, 2016, p. 49).

El documento continúa diciendo:

En el Cuzco en diecinueve días del mes [f.160] de junio de mil y quinientos ochenta, y sinco años: El ilustre Señor Cristóbal de Bocanegra, Teniente de Corregidor y Justicia Mayor de esta ciudad, y su tierra por su Magestad. Visto lo pedido por los Yngas sobre su exepción, y lo probado y presentado por ellos [...] Dijo ... se les guarde dicha exepción y libertad de no servir en servicios [f. 160v.] perzonales a los Yngas contenidos y nombrados en las memorias dadas en esta causa. [...] (De la Puente, 2016, p. 50). Los contenidos en la Memoria, textualmente son los siguientes: Los hijos de Don Cristóbal Paullo Ynga, hijo de Huayna Ccapac = Don Hernando Pomacapi. = Don Luyis Cuzirimachi $=$ dejó dos hijos varones Don Diego Rimachi y otro que darán el nombre $=$ Don Martín Mangotupa $=$ Don Bartolomé Quispe Atauche $=$ Don Alonso Topa Atao $=$ Don Francisco Cana Topa $=$ Don Francisco Topa Guallpa $=$ Don Cristóval Topa $=$ Don Francisco Chichi Topa muerto dejo un hijo $=$ Don Francisco Poma Capi $=$ Y un hijo de Don Juan Rimachi $=35[\ldots]$ Descendientes de Topa Ynga Yupanqui. Don Gonzalo Amaro. Andrés Topa Yupangui (en la nota aclaratoria se verifica que era Una de las cabezas del Capac Aillu, Sarmiento, 2001:137). Don Gerónimo Tito, Don Alonso Topa Yupanqui, Don Domingo Topayupangui, Don Felipe Topayupangui, Don García Cayotopa (nota aclaratoria dice que descienden de Tupa Ynga Yupangui y pertenecen al ayllu Cayao) (De la Puente, 2016, p. 53).

Del mismo documento tenemos:

descendientes de Viracocha Ynga [...] Martín Orccohuaranca, Cristóbal Rimachi, Pablo Yanque Rimachi, Pablo Yupangui, Esteban Orccoguaranga, [...] Los que están en San Jerónimo, Don García Atao Yupangui Ynga Principal (la nota aclaratoria dice que firmó el poder de los orejones a Cristóbal de Molina (1582), era cacique del aillo Hanancuzco 1599 , descendiente de Viracocha y principal del pueblo de Cayra junto con Alonso Yanqui Rimachi en un documento de 1563) (De la Puente, 2016, p. 56). Siguen siendo mencionados: "Pedro Tito, García Rimachi, Don Juan Guaman, Martín Quispe, Agustín Acostupa, Felipe Yupangui, Juan Coro, Juan Copa, Luis Quispe Pedro Atao Yupangui”36 (De la Puente, 2016, p. 56).

35 En esta relación se hace referencia a Ninan Coro, del ayllu de Huayna Ccapac. Este apellido aún existe actualmente en San Jerónimo.

36 Es mencionado en el Documento $\mathrm{N}^{\circ} 3$ del ACCSA. 


\section{Tierras ancestrales y familias actuales que las poseen}

\section{En los documentos se menciona sectores de tierra tanto de cultivo de maíz, trigo, así como sectores de pastizales y guayllares de la comunidad Sucsu Auccaille, muchas de esas tierras siguen siendo poseídas por comuneros del mismo apellido:}

\section{Cuadro 3. Terrenos mencionados en los documentos coloniales y terrenos actuales}

\begin{tabular}{l} 
Terrenos y fuentes de agua de riego 2017 \\
\hline Riega el sector Pumahuanca. \\
Quillinsayoc Q'ente killa. Manante ubi- \\
cado al pie del cerro Guanacauri. \\
Riega el sector de la parte alta de la \\
Comunidad Sucsu Auccaille. \\
Sectores que riega: \\
Quencha guayco, Llutucalle, Condeña \\
kuchu, Picho guayco, Santa Bárbara, \\
Ccaccapata, Simaccata. Peccoccay pata, \\
Cruz pata, Saño ccata, Cruz Ccata. Duyñu \\
ccata, Quencha pata, Accoyoc moqo. \\
Accoyoc pukru, Cuntur mocco, Santa \\
Rosa, Hatun Ccolparo, Huchuy Ccolparo.
\end{tabular}

Terrenos y riego en documentos coloniales

(Diego Senchi Ruca) recaudos y amparo de las tres fanegadas y media de tierras de sembrar maíz y trigo en el asiento y lugar llamado «Tutuna pampa» confina las tierras de Tuyno y unas dos fanegadas y media de tierras en el asiento y lugar llamado Quencha guaico, - Pata pata de sembrar trigos de Moco quencha, Tucu guaico, Maicha pata, Tancar pata, Tocay pampa, Contay moco, bajan a la capilla de Santa Bárbara que tiene dos topos de tierra llamado «llano-guaico».... «y otras fanegadas de tierras nombradas "Tutura-pampa", una fanegada desde la orilla del río desde allí corre por la parte de arriba, pasan a dos piedras plantadas, algo pequeños y de allí va corre esta otra mojón llamado "Senego puquio" pasan hasta la dicha sanja cercada, de allí los árboles plantados de "Opa tancar" /f.4 v./ corre por la dicha sanja hasta del [sic] río de abajo que tiene en la ciudad de Guatanai estando las dichas tierras del Señor Capitán Don Diego Senche Ruca Ynga Señor principal en favor de los dichos mis partes, por Don Andrés de Villella, — Oidor en Concejo de la Real Audiencia de la ciudad de los Reyes nuestro Señor su Juez visitador General de tierras. Por cédula del Rey mi Señor digo que los dichos mis partes tienen y poseen en la Parroquia de San Gerónimo en el asiento llamado Tutuna pampa una fanegada de tierra de sembrar maíz. y otras dos fanegadas de tierra nombradas Quencha quencha, pata pata guaico y por las cuales de sembrar trigo Moco quencha, Ccata quencha, Toco guaico, Maicha pata, bajan Tancar pata, Racay pata, Contay moco, bajan a la capilla de Santa Barbara de Llano guaico estando las dichas tierras unas del —... Ynga las cuales han tenido y poseído» «tres fanegadas y medio de tierras de sembrar maíz y trigo en el ... - lugar llamados "Llanoguayco", "Paccas", "ccatas" de sembrar trigo, "Ccencha ccencha", "pata pata", "Raccay pata", "Ccontaymoco", y los de ... sembrar "Tancarpata", (manchado) estando las dichas tierras "Ccataguaycos" por que sus tieras de dos fanegadas y otros nombres una fanegada de tierras llamados "Tutuna pampa" debajo del reyno cuentro que son ... del Señor Don Diego Sinchi Roca Ynga, dichos ... del Señor San Jerónimo del ayllu Sucsu Aucaille, por ellos y legítimos nietos herederos de Juan Felipe Francisco Conde de Sinchi Roca Ynga su abuelo, /f.5 v./ $/\rangle^{37}$

Sector Pallpanccay, fuente ...- riega el 1595: «Juan Pichota. Dos topos en Halpa corcona, cuatro topos sector alto de Pumahuanca, donde están de tierra de andenes en Paallpancay que fueron del sol y medio los sectores de Palalpanccay primero, topo en Guayllapampa».

Munay huarmi y Huaca puncu.

37 Título de propiedad de la Comunidad. ACCSA. 
Terrenos y fuentes de agua de riego 2017 Terrenos y riego en documentos coloniales

Captación del río Huanacauri que riega 1595: «Don Juan Guamán. Un topo en Cairapampa. “Juan Ezequiel todo el sector de Cayra pampa, hasta la Topa. Un topo en Cairapampa», Francisco Cari. Un topo en la línea férrea, como Molino pampa, San tercera suerte de Cairapampa,

Miguel, Silla mocco, Pachac huaycco,

Duyñu k'uchu, Huerta pata, Riel pampa.

Fuente elaboración propia a partir de títulos de propiedad de la comunidad y padrón de riego.

\section{Cuadro 4. Terrenos mencionados en documentos coloniales y sus propietarios actuales que mantienen el apellido de los propietarios que aparecen en documentos coloniales}

\begin{tabular}{|c|c|}
\hline Terrenos mencionados en documentos coloniales y propietarios. & $\begin{array}{l}\text { Terrenos actuales y usuarios } \\
\text { actuales } 2017\end{array}$ \\
\hline \multicolumn{2}{|l|}{$\begin{array}{l}1544 \text { Cédula de reconocimiento a Sinche Ruca Tito YupanquiYnga, dada } \\
\text { por el Rey }\end{array}$} \\
\hline $\begin{array}{l}1630 \text { «Don García Topa Atauche y Don Pedro Antón Atayupanqui y Don } \\
\text { Sebastián Poma Supa Yngas, caciques y gobernadores de los ayllus Sucsu y } \\
\text { Auccaile de la generación de Viracochán Ynga, Yaguar Vacac Ynga Señores } \\
\text { que fueron de esta tierra rreducido en la Parroquia de San Jerónimo tierras } \\
\text { de la rrepartición que tienen poseen en los asientos de, Cayrapampa (5), } \\
\text { Condenauqui (2), Colparu (1) pampa, Palpamcay pampa, Guacapongo, } \\
\text { Picocaipampa (4), Cozcopampa, Copampa y Quinchacaira (3), Tinyacucho, } \\
\text { Colpampa, Chuncuran yami, Cullulaqta, Siusacucha, Totora villa, Poma- } \\
\text { guanca, que están asentadas en el libro de la dicha rrepartición». Documento } \\
\text { 1./ 1656 Don Pascual Atayupanqui y Domingo Ramos las mismas tierras. }\end{array}$ & $\begin{array}{l}\text { Atayupanqui } \\
\text { Puma } \\
\text { Supha } \\
\text { Ramos Sinchi Roca }\end{array}$ \\
\hline $\begin{array}{l}\text { Sector Ccolparo grande }{ }^{38} \\
\text { Cacique Don Pedro Antón Atayupanqui 1595, Don Juan Atayupanqui 1595, } \\
\text { Don García Ataoyupangui } 1691\end{array}$ & $\begin{array}{l}\text { (1) Sector Ccolparo grande }{ }^{39} \\
\text { Orrccon Tito } \\
\text { Atayupanqui }\end{array}$ \\
\hline $\begin{array}{l}\text { Sector Ccolparo chico } \\
\text { Don García Topa Atauche y Don Pedro Antón Atayupanqui y Don Sebastián } \\
\text { Poma Supa Yngas. En 1595: «Don Alonso Tito Atauche un topo de Pall- } \\
\text { panccay. Un topo en la segunda suerte de Cairapampa, Uno y medio topo en } \\
\text { Guaca pongo, que a derecho ser suyo y heredero del Guayna Capa por ser } \\
\text { su bis nieto, un topo en Collparo huqui, un topo en Picoraipampa, un topo } \\
\text { en Uscollopampa, un topo en Copco pampa, medio topo en Guayllapampa, } \\
\text { un topo en Oscollopampa». } \\
\text { 1595: «Juan Ttito. Un topo en Collparo, un topo en Quillairo, un topo en } \\
\text { Pomaorcco, un topo en Copco pampa». }\end{array}$ & $\begin{array}{l}\text { Sector Ccolparo chico } \\
\text { Atayupanqui Tito } \\
\text { Usca Rimachi } \\
\text { Sutta Raura } \\
\text { Quispe } \\
\text { Ttito }\end{array}$ \\
\hline $\begin{array}{l}\text { Sector Condeña C'ucho } \\
\text { Don García Topa Atauche y Don Pedro Antón Atayupanqui y Don Sebastián } \\
\text { Poma Supa Yngas }\end{array}$ & $\begin{array}{l}\text { (2) Sector Condeña C'uchu } \\
\text { Atayupanqui } \\
\text { Quispe }\end{array}$ \\
\hline Sector Quishuar pata & $\begin{array}{l}\text { Sector Quishuar pata } \\
\text { Atayupanqui }\end{array}$ \\
\hline
\end{tabular}

38 Pusimos la numeración para comparar los nombres de tierras antiguos con los actuales.

39 Pusimos la numeración para comparar los nombres de tierras antiguos con los actuales. 
Terrenos mencionados en documentos coloniales y propietarios.

\begin{tabular}{|c|c|}
\hline $\begin{array}{l}\text { Quencha huayco } \\
\text { Don García Topa Atauche y Don Pedro Antón Atayupanqui y Don Sebastián } \\
\text { Poma Supa Yngas }\end{array}$ & $\begin{array}{l}\text { (3) Quencha huayco } \\
\text { Sinchi Roca } \\
\text { Atayupanqui } \\
\text { Sutta } \\
\text { Rimachi }\end{array}$ \\
\hline Sector Santa Bárbara & $\begin{array}{l}\text { Sector Santa Bárbara } \\
\text { Atayupanqui } \\
\text { Quispe } \\
\text { Sinchi Roca Tupac Yupanqui }\end{array}$ \\
\hline Sector Ccacca pata & $\begin{array}{l}\text { Sector Ccacca pata } \\
\text { Sinchi Roca Tupac Yupanqui } \\
\text { Valderrama Sinchi Roca }\end{array}$ \\
\hline $\begin{array}{l}\text { Sector Peccoccay pata } \\
\text { Don García Topa Atauche y Don Pedro Antón Atayupanqui y Don Sebastián } \\
\text { Poma Supa Yngas } \\
\text { 1595: «Don Juan Guamán. Un topo en Cairapampa, un topo en la tercera } \\
\text { suerte de Collparo, un topo en Picoraipampa, un topo en Cumillunca guaico, } \\
\text { un topo en Allpahurcuna». }\end{array}$ & $\begin{array}{l}\text { (4) Sector Peccoccay pata } \\
\text { Atayupanqui } \\
\text { Atayupanqui Tito } \\
\text { Sinchi Roca Tupac Yupanqui } \\
\text { Quispe } \\
\text { Paucar Apumayta } \\
\text { Guamán }\end{array}$ \\
\hline Sector Sañu C'jata & $\begin{array}{l}\text { Sector Sañu C'jata } \\
\text { Quispe Atayupanqui } \\
\text { Rimachi }\end{array}$ \\
\hline Sector Dueño C'jata & $\begin{array}{l}\text { Sector Dueño C'jata } \\
\text { Atayupanqui } \\
\text { Quispe }\end{array}$ \\
\hline $\begin{array}{l}\text { Sector Q'encha C'jata } \\
\text { Don García Topa Atauche y Don Pedro Antón Atayupanqui y Don Sebastián } \\
\text { Poma Supa Yngas }\end{array}$ & $\begin{array}{l}\text { (2) Sector Q'encha C'jata } \\
\text { Atayupanqui }\end{array}$ \\
\hline $\begin{array}{l}\text { Sector Llutu calla } \\
\text { 1595: «Miguel Quisso un topo en Pallpancai, un topo en Quillairo, un topo } \\
\text { en Llutocalla, un topo en Copco pampa». }\end{array}$ & $\begin{array}{l}\text { Sector Llutu calle } \\
\text { Atayupanqui } \\
\text { Ttito }\end{array}$ \\
\hline Sector Accoyocmocco & $\begin{array}{l}\text { Sector Accoyoc mocco, Ata- } \\
\text { yupanqui }\end{array}$ \\
\hline Sector Accoyoc pucro & $\begin{array}{l}\text { Sector Accoyoc pucro, Ata- } \\
\text { yupanqui }\end{array}$ \\
\hline Sector Kuntur mocco & $\begin{array}{l}\text { Sector kuntur mocco, Atayu- } \\
\text { panqui, Quispe Ttito } \\
\text { Ttito }\end{array}$ \\
\hline Sector Huanacauri & $\begin{array}{l}\text { Sector Huanacauri, Atayupan- } \\
\text { qui, Quispe, Ttito }\end{array}$ \\
\hline Sector Molino pampa & $\begin{array}{l}\text { Sector Molino pampa, Tupac } \\
\text { Yupanqui } \\
\text { Rimachi, Ttito }\end{array}$ \\
\hline
\end{tabular}


Terrenos mencionados en documentos coloniales y propietarios.

Sector K'ayra pampa

1595: «Don Juan Guamán. Un topo en Cairapampa, un topo en Cumillunca guaico, un topo en allpahurcuna». «Juan Ezequiel Topa. Un topo en Cairapampa, un topo en Collparo, un topo en Quillairo, un topo en Copco pampa». 1595: «Francisco Cari. Un topo en la tercera suerte de Cairapampa».

Sector Silla mocco

Sector Dueño pampa

Sector Dueño K’uchu

Sector Pallpanccay (no está en padrón)

1595: «Juan Pichota. Dos topos en Halpa corcona, cuatro topos de tierra de andenes en Paallpancay que fueron del sol y medio topo en Guayllapampa». 1595: «Martín Quispe. Pallpancai, otro de los indios Auccaillis, y un topo en Copco pampa».

1595: «Juan Quesso Yupanqui medio topo».

«Gerónimo Puso mayo. 1 topo en Pallpanccay».

Sector Quillairu (no está en padrón actual)

1595: «Francisco Quesso. Un topo en Pecoraypampa, un topo en Quillairo pampa, un topo en Usccollo pampa. Domingo Atao. un topo en Quillairo, un topo en Copco pampa».

Pedro Ataoyupanqui, un topo en Quillairo,

Inés Toctto un topo en Quillairo".Ff-52:

Aca mana pampa.- «Posesión de las tierras del asiento de Aca mana pampa que lindan con Apumaita y Vicaquirao y por la otra Sañoc y Tarpuntay y Culluncas orco» (Doc. 1595)
Terrenos actuales y usuarios actuales 2017

(5) Sector K'ayra pampa

Guamán o Huaman

Atayupanqui

Ttito

Tupac Yupanqui

Sector Sillamocco, Orccohuarancca, Ttito

Atayupanqui, Quispe

Sector Dueño pampa, Atayupanqui

Sector Dueño K'uchu, Atayupanqui, Ttito

Sector Pallpanccay

Sector Quillairo

En la actualidad ya no aparece como sector de esta comunidad.

Fuente: Elaboración propia a partir del plano de la Comisión de Regantes Comunidad Sucsu Auccaille y Título de 1595 .

En los cinco documentos se menciona a los representantes temporales del aillo Sucsu Auccaille como sigue:

En la visita de don Francisco de Loayza, juez visitador de 1595, se menciona a don Pascual, alcalde y cacique de los indios de Saylla. En el mismo documento se menciona a don Sebastián Suta, don Sebastián Maita. Aparecen como principales de la Parroquia del Señor San Jerónimo, como quienes firmaron y dieron fe al Auto de Sucsu San Jerónimo, cuando el auto fue pregonado y divulgado. En el documento de provisión de 1630 sobre los ayllos de Sucso Auccaille de la Parroquia de San Jerónimo como Caciques de los indios de Sucsu Auccaille, don Pedro Antón Atayupanqui, don Gregorio Tupa Atauche, caciques de los indios de Sucsu y Auccaille. En el mismo documento aparece don Sebastián Poma Supa. Yngas y caciques y gobernadores de los aillos de Sucsu Auccaille de la Parroquia de San Jerónimo. Como testigos de 
dicha provisión aparecen don Tomás Tito Aatauche, don Pedro Paro Tupa, don Gabriel Inca Tito, principales de dicha parroquia de San Jerónimo.

En el mismo documento de concordancia, los caciques de Sucso Auccaille aparecen don Andrés López y don Juan Atayupanqui, caciques de dichos aillos.

En el documento de 1691 de Corregimiento 25- 1691 -92 (f.38 al 42) sobre «los indios de la parroquia de San Jerónimo con Don Guillermo Valladares sobre unas tierras». El documento presenta como testimonio de la repartición de las tierras y visita que hizo el capitán don Francisco de Loayza. Aparecen como caciques don Alonso Tito Atauche, heredero de Huayna Ccapac por ser su nieto.

En el documento distribución y acomodamiento que hace el capitán, aparecen como caciques don García Ataoyupangui, cabeza y cacique principal de dicha Parroquia, don Pedro Orccoguaranga, mandón de este aillo y alcalde ordinario. En el mismo documento aparece Juan Pichota como dueño de dos topos en Huallpa Carcona - Paallpancay que fuera tierra del sol.

En documento de 1695 sobre «tierras del Señor Don Diego Senchi Ruca Ynga Cacique Gobernador de la ciudad del Cusco de la Parroquia de San Jerónimo, del aillo Sucsu Auccaille, que fue nieto legítimo heredero de las dichas tierras contenidas en esta real provisión del Rey mi Señor». También anota «hijos, nietos legítimos de Juan Felipe Francisco Conde Ynga Senchi Ruca [f.4] Ynga Capac que fue su bisabuelo materno alcanzó desde España un decreto del Señor Rey Don Carlos Quinto».

En el mismo documento aparecen como caciques Pascual Tupa Yupanqui panaca y Pascual Quispe Rimachi, en el inicio de construcción del templo de San Jerónimo, 1571.

En documento de 1701 de Corregimiento /27/1700-11.24/otc./1701, aparece como cacique Gerónimo Cusi Rimachi Luna, cacique principal de la Parroquia San Jerónimo don Juan Orccoguaranga Cacique del Aillo Sucsu Auccaille.

\section{Panacas: supervivencia y continuidades}

La tierra, la de cultivo y la de pastizales; el agua de consumo y de riego; son los vínculos más importantes que anclan a las familias de Sucsu Auccaille y a sus descendientes en todos los tiempos, en esta localidad. Lo interesante es que lo que une desde esa época a Auccaille y a Sucsu no solo es que descienden de Yaguar Huacac, sino que ambos son considerados del Anti suyu. Hablando del distrito de San Jerónimo, existen descendientes de las once panacas consideradas por Sarmiento de Gamboa y mantienen sus apellidos: 


\section{Chima: Sisa}

2. Raurahua: Sinchi Roca,

3. Hahuainñin: Tito Condemaita, Tisoc

4. Uscamaita: Ushca, Tambo Uscamaita, Paucarmaita, Maita.

5. Apumaita: Apumaita. Cusiguaman, Cocamaita.

6. Huekaquirau: Inca Roca, Guallpa.

7. Aucailli: Guamanrimachi, Rimachi.

8. Sucsu: Atau Yupanqui. García Atau yupangui, Chalco Yupangui, y Tito.

9. Iñaca: y Hatun ayllu: Pachacuti,

10. Ccapac: Tupayupanqui, Yupanqui.

Cuadro 5. Comparación de apellidos de descendientes de Viracocha y Yahuar Huacac a inicios de la Colonia con los apellidos actuales de los comuneros de Sucsu Aucaille

\begin{tabular}{|c|c|}
\hline Descendientes de Viracocha Inca y Yahuar Huacac & $\begin{array}{l}\text { Apellidos que se mantienen } \\
\text { actualmente }\end{array}$ \\
\hline $\begin{array}{l}\text { «descendientes de Viracocha Ynga ... Martín Orccohuaranca, Cristóbal } \\
\text { Rimachi, Pablo Yanque Rimachi, Pablo Yupangui, Esteban Orccogua- } \\
\text { ranga, [... Los que están en San Jerónimo, Don García Atao Yupangui } \\
\text { Ynga Principal (la nota aclaratoria dice que firmó el poder de los } \\
\text { orejones a Cristóbal de Molina (1582), era cacique del aillo Hanan- } \\
\text { cuzco 1599, descendiente de Viracocha y principal del pueblo de Cayra } \\
\text { junto con Alonso Yanqui Rimachi en un documento de 1563)» (De la } \\
\text { Puente, 2017, p. 58). Siguen siendo mencionados: «Pedro Tito, García } \\
\text { Rimachi, Don Juan Guaman, Martín Quispe, Agustín Acostupa, Felipe } \\
\text { Yupangui, Juan Coro, Juan Copa, Luis Quispe Pedro Atao Yupangui» } \\
\text { (De la Puente, 2016, p. 58). }\end{array}$ & $\begin{array}{l}\text { Atau Yupanqui } \\
\text { Chalco Yupangui } \\
\text { Tito. } \\
\text { Quispe, } \\
\text { Guamán, } \\
\text { Yupanqui. } \\
\text { Yanqui Rimachi, } \\
\text { Rimmachi }\end{array}$ \\
\hline $\begin{array}{l}\text { «Yaguarguacac Ynga Yupangui que fue señor y rei desta tierra del } \\
\text { qual subcedieron los Yngas del aillo Aucaille Panaca y del dicho aillo } \\
\text { conocen al presente a Hernando Tito y a Don Pedro Palomino y a Don } \\
\text { Martín Chara» (De la Puente, 2016, p. 61). }\end{array}$ & $\begin{array}{l}\text { Tito, } \\
\text { Chara, } \\
\text { Rimachi, } \\
\text { Guamanrimachi. }\end{array}$ \\
\hline
\end{tabular}

Fuente: Elaboración propia a partir de documentos del Archivo de la comunidad Sucsu Auccaille.

A partir de la entrada de los españoles al Cusco en 1533 se produce un gran caos y destrucción. Además de la guerra existente entre partidarios de Huáscar y partidarios de Atahuallpa, se suceden guerras entre los españoles, entre los incas y los españoles, y los incas tomaban uno y otro partido, como hemos visto, viviendo en la cotidianeidad la destrucción del Estado Incaico. El documento de 1595 es el de tratar de poner orden — colonial — frente al colapso organizacional producto de la conquista. En ese año los descendientes de incas mencionados tenían mayores cantidades de tierra, que los comuneros descendientes de hatun runas (hombres del pueblo). 
A lo largo de los años la comunidad pierde territorio, recuperando una pequeña parte en la época de la Reforma Agraria, en 1970. Lo interesante es que mantienen su propiedad sobre sus tierras y mantienen una continuidad de organizar su producción y sus intercambios. Un grupo social que mantiene un control sobre sus organización económica y social internos, no llegan a ser diluidos ni liquidados culturalmente por los conquistadores, colonizadores. Si bien hay fuertes cambios también tienen continuidades. Sucsu Auccaille a través del tiempo muestra su capacidad para negociar, su destreza para sobrevirvir. Para traducir culturalmente legislación colonial y poder continuar. En la región del Cusco (y aún en una zona mas extensa), en general, somos descendientes culturales de la etnia Inca. En San Sebastián y San Jerónimo ${ }^{40}$, existen descendientes de Incas y que llevan los denominativos de determinados Incas que fueron gobernantes del Estado del Tahuantinsuyo. Como muestra la documentación de la Comunidad de Sucsu Auccaille, descienden de incas y poseen terrenos y agua de riego que fue de los incas. En este sentido, efectivamente, existe supervivencia y continuidad, tanto del grupo social como de la cultura inca. En 1571, en que se pone la primera piedra para la construcción del Templo de San Jerónimo, los incas participan en la construcción del nuevo orden colonial, como intermediarios entre sus ayllus y las autoridades coloniales, son denominados «caciques», se adaptan al nuevo orden, y a usar las nuevas leyes buscando defender sus tierras, defender sus familias, sus ayllus. Generación de guerreros que se vio entre la caída del gran Estado del Tahuantinsuyo, la guerra entre Huascar y Atahuallpa y después de la masacre de la conquista, tomar partido entre los batallones en la guerra entre pizarristas y almagristas, y siempre dirigiendo batallones de su propia gente, participando en las batallas entre el rebelde Gonzalo Pizarro y el pacificador La Gasca. Toda una vida guerreando, sobreviviendo y tratando de construir un mundo más seguro para sus descendientes, pedían al rey de España reconocerles sus servicios y reconocer que los ganaban para sus hijos e hijas y sus vástagos, que hoy conforman la comunidad campesina Sucsu-Auccaille.

40 En San Jerónimo, las calles llevaban los nombres de ayllus y panacas Incas: Aqa mana, Awqaylli, Orqon Apu mayta, Sucsu, Qollana, Sorama, Ccaqsamunati, Anawarqe, Raw raw, y Andamachay Ushcamayta, que era una huaca ubicada en el actual altar mayor del templo de San Jerónimo. 


\section{ARCHIVOS}

Archivo de la Comunidad Campesina Sucsu Auccaille de San Jerónimo, Cusco

Archivo Regional del Cusco

Archivo Privado Familiar de Octavio Sinchi Roca

\section{REFERENCIAS}

De la Puente Luna, José Carlos (2016). Incas, pecheros y caballeros hidalgos: la desintegración del orden incaico y la génesis de la nobleza incaica colonial en el Cusco del siglo XVI. Revista Andina, 54.

Elward, Ronald (16 de septiembre, 2012). Los Sinchi Roca: el poderoso vínculo con la tierra. El Comercio, p. A22.

Hernández Astete, Francisco (2008). Las panacas y el poder en el Tahuantinsuyo. Bulletin de l'Institut français d'études andines, 37(1), 29-45.

Rostworowski, María (1983). Estructuras andinas del poder: ideología religiosa y política. Lima: IEP.

Uhle, Max (1969). Estudios sobre la historia incaica. Lima: Universidad Nacional Mayor de San Marcos.

Zuidema, Tom (1974/76). La imagen del Sol y la Huaca de Susurpuquio en el sistema astronómico de los Incas en el Cuzco. Journal de la Société des Américanistes, 63, 199-230.

Zuidema, Tom (2002). La organización religiosa del sistema de panacas y memoria en el Cuzco incaico. Cuzco: CBC. 